\title{
La enseñanza de la programación. Una experiencia en la formación de profesores de Informática
}

\author{
KEILA IRENE DÍAZ TEJERA* \\ EMMA FIERRO MARTÍN** \\ MARÍA AMELIA MUŃOZ PENTÓN ${ }^{* * *}$ \\ Universidad Central Marta Abreu de las Villas - Santa Clara - Cuba
}

Recibido el 13-03-2017; primera evaluación el 06-06-2018; segunda evaluación el 12-06-2018; tercera evaluación el 26-06-2018; aceptado el 27-06-2018

\section{RESUMEN}

La enseñanza de la programación tiene, entre sus objetivos esenciales, la formación y desarrollo de habilidades, por parte de los estudiantes, que posibiliten la resolución de problemas del ámbito escolar, profesional o de la vida práctica, teniendo en cuenta los recursos que brindan los diferentes lenguajes de programación. En la formación de profesores de Informática, este proceso posee características que lo distinguen. El profesor en formación debe desarrollar la habilidad y, a la vez, apropiarse de los procedimientos necesarios para poder dirigir este proceso

\footnotetext{
* Profesor titular. Doctor en Ciencias Pedagógicas. Máster en Nuevas Tecnología para la Educación. Licenciada en Educación especialidad Matemática. Profesora Principal de Año Académico de la carrera Licenciatura en Educación Informática. Jefa de las disciplinas Lenguajes y Técnicas de Programación y Sistemas de Aplicación. Miembro de la Sociedad Cubana de Matemática y Computación. Miembro de la Asociación de Pedagogos de Cuba. Correo electrónico: keilad@uclv.cu

" Profesor auxiliar. Doctor en Ciencias Pedagógicas. Máster en Nuevas Tecnologías para la Educación. Licenciada en educación. Especialidad Matemática. Jefa de Departamento Educación Laboral Informática en Universidad Central de Las Villas «Marta Abreu». Profesora de Lenguaje y Técnica de Programación. Profesora de las TIC en la gestión de la información. Miembro de la Asociación de Pedagogos de Cuba. Correo electrónico: efierro@uclv.cu

${ }^{* * *}$ Profesor titular. Doctor en Ciencias Pedagógicas. Máster en Matemática Aplicada. Licenciada en educación. Especialidad Matemática-Física. Jefa de proyecto de investigación. Coordinadora de la carrera Licenciatura en Educación. Informática en Universidad Central de Las Villas «Marta Abreu». Miembro del colectivo nacional de la disciplina Lenguajes y Técnicas de Programación para las carreras Informática y Educación Laboral Informática. Miembro de la Comisión nacional de la carrera Licenciatura en Educación Informática. Presidenta de la Delegación de la Sociedad Cubana de Matemática y Computación en la provincia Villa Clara. Miembro de la Asociación de Pedagogos de Cuba. Correo electrónico: mmpenton@uclv.cu
} 
en la escuela. En este artículo se exponen los principales resultados obtenidos al respecto durante el proceso de enseñanza aprendizaje de varias asignaturas de Programación en la formación de profesores de Informática con la sistematización de experiencias vividas durante nueve ańos.

Palabras clave: habilidades, informática, proceso enseñanza aprendizaje, formación de profesores

\section{The teaching of programming: An experience in the training of Informatics teachers}

\section{Abstract}

The education of programming has between its main objectives, the development and the formation of skills on the part of the students, who make possible the resolution of problems of the school, professional or of the practical life taking into account the different resources that offer the languages of programming. In the training course of computer teachers, this process has some characteristics that distinguish it. The teachers to be must develop the skill and at the same time to appropriate of the necessary procedures to direct this process in the school. This article presents the main results obtained regarding this topic during the teaching learning process of several subjects of Programming in the training of Informatics teachers, with the systematization of living experiences, during nine years.

Keywords: skill, Informatics, teaching learning process, teacher training

\section{O ensino da programaçáo. Uma experiência na formaçáo de professores da Informática}

ResUmo

$\mathrm{O}$ ensino da programação, tem entre seus objectivos essenciais a formação e desenvolvimento de habilidades por parte dos estudantes que tornem possível a resoluçấo de problemas do âmbito escolar, profissional ou da vida práctica, tendo em conta os recursos que brindam as diferentes linguagens de programação. $\mathrm{Na}$ formação de professores de Informática, este processo possui características que o distinguem. O professor em formação deve desenvolver a habilidade e, ao mesmo tempo, apropiar - se dos procedimentos necessários para poder dirigir este processo na escola. Neste artigo se expóem os principiáis resultados obtidos a respeito durante o processo de ensino aprendizagem de várias disciplinas de Programação na formação de professores de informatica, com a sistematização de experiências vividas, durante nove anos.

Palavras-chave: habilidade, Informática, processo ensino aprendizagem, formação de professores 


\section{INTRODUCCIÓN}

El objetivo principal de la enseñanza de la programación en la formación inicial del profesor de Informática es resolver problemas, con el empleo de un lenguaje de programación. Para darle cumplimiento, se necesita que el estudiante desarrolle el sistema de habilidades específicas de la programación y se apropie del sistema de conocimientos y procedimientos en correspondencia con las técnicas de programación y las herramientas de desarrollo en estudio. Sin embargo, el modelo de profesional de la especialidad demanda, además, que el profesor en formación sea capaz de asimilar modos de actuación que le faciliten su futuro desempeño en la escuela.

La experiencia práctica del colectivo de profesores que imparten esta materia en la Universidad Central Marta Abreu de las Villas, así como los resultados de investigaciones realizadas sobre el proceso de enseñanza aprendizaje de la programación, han demostrado insuficiencias en el proceso de formación y desarrollo de la habilidad para resolver problemas con el empleo de un lenguaje de programación, así como en la adquisición consciente de las acciones que la componen. Entre las causas detectadas, se muestran las carencias que poseen los estudiantes en la realización de dichas acciones, que no son más que habilidades específicas de la programación. Se revela también, entre las causas de las insuficiencias, que los profesores no incorporan, en el proceso de enseñanza aprendizaje, métodos que permitan, al futuro profesor, conocer los procederes metodológicos que puede emplear para dirigir el proceso de formación y desarrollo de las habilidades específicas de la programación en su accionar en la escuela.

Es innegable que el proceso de formación y desarrollo de habilidades posee características generales aplicables a cualquier disciplina. Sin embargo, existen otras particularidades, tanto de la enseñanza de la programación como de la formación de profesores, que también deben tomarse en consideración al dirigir este proceso. El presente trabajo pretende mostrar los resultados alcanzados en la temática, con la sistematización de las experiencias vividas por las autoras durante nueve años en la dirección del proceso de formación y desarrollo de habilidades informáticas de la programación. La sistematización se describe en tres etapas establecidas atendiendo a los cambios curriculares ocurridos en los modelos de formación de los estudiantes de la especialidad de Informática durante los nueve ańos. En cada una de las etapas se describen tres momentos esenciales: el diseńo, la recuperación histórica e interpretación crítica de las experiencias y la elaboración de los productos de comunicación de las experiencias sistematizadas. Para la recopilación de la información 
fueron empleados varios métodos y técnicas investigativas. Entre ellos, se destacan el análisis documental, el diario de campo, la observación, el análisis de los productos del proceso pedagógico y las entrevistas. Se empleó, además, la triangulación de fuentes para contrastar la información obtenida.

\section{MarCo teórico}

En la formación inicial de profesores, el proceso de enseñanza aprendizaje constituye la vía fundamental para la adquisición de conocimientos, procedimientos, habilidades, normas de comportamiento y valores que le permitan al estudiante enfrentar su labor como profesor en cualquier nivel de educación. El reto se centra en enseñar al futuro profesor, además de los contenidos específicos de la ciencia en la que se forma, la pedagogía y la didáctica, «el aprender a pensar, identificar sus procesos, descubrir sus errores, formular juicios, adoptar decisiones para actuar con independencia y libertad personal» (Coloma y Tafur, 2000, p. 56).

Varios son los objetivos que se formulan en la formación de profesores para lograr alcanzar esta aspiración. Dentro de ellos se encuentra la formación de habilidades.

La habilidad es sinónimo de saber hacer y permite al ser humano realizar acciones cada vez más perfectas de forma racional. Reed (2007), citado por Báez y Onrubia (2016), caracteriza las habilidades como «rutinas cognitivas existentes y empleadas para facilitar la adquisición y producción del conocimiento» (p. 96), por lo que pondera solamente la habilidad como un componente del contenido. Sin embargo, para esta investigación, se precisa caracterizar la habilidad de manera integral analizándola tanto desde el punto de vista psicológico, como didáctico. Por tal razón, se asume el criterio de Álvarez (1992), quién define la habilidad como "la dimensión del contenido que muestra el comportamiento del hombre en una rama del saber propio de la cultura de la humanidad. Es, desde el punto de vista psicológico, el sistema de acciones y operaciones dominado por el sujeto que responde a un objetivo» (p. 69).

El estudio teórico realizado lleva a las autoras a afirmar que, para formar adecuadamente una habilidad, es preciso estructurar pedagógicamente los pasos a seguir, en correspondencia con las características que debe lograr la acción para devenir en habilidad. De igual manera, las autoras comparten el criterio de López (1990) al considerar que, en el proceso de adquisición de una habilidad se deben precisar dos etapas: la de formación de la habilidad y la de su desarrollo. La formación de la habilidad comprende la adquisición consciente de los modos de actuar, cuando, bajo la guía del profesor, el estudiante 
recibe la orientación adecuada sobre la forma de proceder. Esta etapa es fundamental para la adquisición de una habilidad, sin embargo, no se le debe restar importancia a la etapa del desarrollo pues es en ella donde «una vez adquiridos los modos de acción, se debe iniciar el proceso de ejercitación; o sea, se debe comenzar a usar la habilidad recién formada en la cantidad necesaria y con la frecuencia adecuada, de modo que vaya haciéndose más fácil de reproducir y se eliminen los errores» (López, 1990, p. 3).

En la enseñanza de la Informática, ha sido recurrente el estudio del proceso de formación y desarrollo de las habilidades. Se destacan las investigaciones realizadas por Alea, Miqueo y Aguiar (2005), Díaz y Crespo (2010) y Expósito et. al. (2001) quienes demuestran la existencia de una tendencia a circunscribir las habilidades informáticas a la manipulación de software, ya sean de carácter general o específicos. Sin embargo, la Informática cuenta con disciplinas en las que no basta solamente desarrollar habilidades en la manipulación de software, como el campo de la programación.

El objetivo esencial de la programación es la utilización de las computadoras con el fin de resolver problemas, a través de los recursos que brindan los diferentes lenguajes de programación. Para darle cumplimiento, es preciso que, además de manipular los software relacionados con la disciplina, el estudiante domine habilidades informáticas específicas de la programación. Al profundizar en la temática, Díaz (2013) corrobora que no existe unanimidad en la denominación y estructura de las habilidades informáticas específicas que debe adquirir el estudiante en el proceso de enseńanza aprendizaje de esta disciplina. Por tal razón, propone un sistema de habilidades que toma en consideración las similitudes encontradas en los estudios realizados por autores de diferentes países, entre los que se destacan Fischer (2011), Sánchez (2007), Suárez (2008) y Vasconcelos (2007). El sistema de habilidades informáticas de la programación propuesto por Díaz (2013) es asumido por las autoras para el desarrollo de esta investigación, por considerarlo integrador y en correspondencia con los objetivos de la formación de profesores de Informática en Cuba.

El análisis de los estudios realizados por los autores anteriormente mencionados, así como de los trabajos presentados por Aguilasocho (2004), Ferreira y Rojo (2006), Jackson y Miller (2009), Matthiasdóttir (2011) y Murillo (2006), permite apreciar el reconocimiento de la resolución de problemas como vía fundamental para el desarrollo de habilidades de la programación a nivel internacional. El empleo de métodos problémicos que conduzcan al estudiante a enfrentarse al sistema de acciones y operaciones necesarias, para darle solución a una problemática dada, ofrece la posibilidad de enseñarle cuáles son las acciones necesarias y suficientes que debe realizar para analizar las situaciones 
problémicas surgidas, elaborar algoritmos que permitan darle solución, codificarlos en correspondencia con el lenguaje de programación que estudie e implementarlo de manera eficiente y óptima en el entorno de desarrollo seleccionado, teniendo en cuenta el paradigma de la programación que se emplee.

Al utilizar los métodos problémicos, en el proceso de formación y desarrollo de habilidades informáticas de la programación, las autoras consideran que, en la fase de formación de la habilidad, debe predominar el método de enseñanza explicativo motivador. Con su empleo, el profesor, a partir del planteamiento de una situación problémica, le brinda al estudiante una base orientadora completa y generalizada sobre la habilidad que requiere formar para solucionarla. De esta manera, el estudiante, a partir de las explicaciones dadas y producto de su actividad independiente, puede lograr darle solución.

La fase de desarrollo de la habilidad debe comenzar con el empleo del método de enseñanza explicativo motivador e ir introduciendo paulatinamente el método motivador. El profesor debe plantear preguntas y tareas problémicas para organizar la actividad independiente que lleven al estudiante a descubrir de modo independiente las acciones de las habilidades informáticas de la programación que le permitan darle solución, llegando, incluso, a plantearse nuevos problemas y buscar sus propias vías para resolverlos.

Resulta significativo, además, durante el proceso de formación y desarrollo de habilidades de la programación, el empleo de estrategias didácticas, conocidas como:

[...] un conjunto de acciones, ordenadas y secuenciadas conscientemente por el docente, con un propósito o intencionalidad pedagógica determinado, responden a decisiones pertinentes basadas en una reflexión sobre la mejora del proceso de enseñanza y aprendizaje; y sus elementos dependen de la subjetividad, los recursos existentes y del contexto donde se desarrollan. Dentro del conjunto de estas acciones planificadas, no se descarta el uso de métodos, técnicas y procedimientos que contribuyan a la optimización del proceso de enseñanza y aprendizaje. (Vargas, 2013a), citada en Vargas (2014, p. 28).

A partir de esta definición y tomando como criterio la existencia de una gran variedad de tipos de estrategias didácticas, Vargas (2014) propone cuatro tipos para el desarrollo de la identidad cultural en educación primaria:

- Estrategias para generar o activar conocimientos previos.

- Estrategias para facilitar la adquisición del conocimiento.

- Estrategias para el desarrollo de contenidos procedimentales, habilidades cognitivas y habilidades psicomotrices.

- Estrategias para la adquisición de actitudes, valores y normas 
El análisis de la caracterización de estos tipos de estrategias didácticas lleva a las autoras a plantear que, para la adquisición de habilidades de programación en la formación de profesores de Informática, también pueden diseñarse estrategias que se correspondan con estos mismos tipos. Sería necesario, solamente, emplear técnicas y procedimientos en correspondencia con los objetivos que se deseen alcanzar.

Durante el proceso de formación y desarrollo de habilidades informáticas de la programación, también resulta importante el empleo de medios de enseńanza. Los tradicionales (pizarra, materiales impresos, láminas) permiten al estudiante el desarrollo de habilidades lógicas y específicas de la programación, vinculadas con la habilidad informática general para resolver problemas con computadoras. Entre ellos, se destacan el análisis del problema, la identificación de conceptos y procedimientos a emplear y la descripción de los pasos principales para elaborar su solución. Mientras que la utilización de la computadora y, en especial, de los software seleccionados como objeto de estudio, además de contribuir a desarrollar las habilidades antes mencionadas, también facilitan el desarrollo de otras habilidades específicas de la programación, que solo pueden lograrse con la interacción entre el estudiante y un entorno de desarrollo integrado.

A partir de estos elementos generales acerca del proceso de formación y desarrollo de habilidades informáticas de la programación, se presenta a continuación la sistematización de las experiencias vividas por las autoras en la formación inicial del profesor de Informática.

\section{Metodología}

Como estrategia general, en el trabajo, se revela la sistematización de las experiencias vividas por las autoras durante nueve años en la dirección del proceso de enseñanza aprendizaje de la programación. La sistematización ha sido analizada y empleada en la investigación educativa por diversos autores, entre ellos Fierro (2016), Navarro y Roche (2013) y Van de Velde (2008). De una forma u otra, los autores consultados coinciden en que la sistematización es un proceso de recuperación y apropiación de una práctica formativa determinada que, al relacionar sistémica e históricamente sus componentes teórico-prácticos, permite a los sujetos comprender y explicar los contextos, sentidos, fundamentos, lógicas y aspectos problemáticos que presenta la experiencia.

Las autoras asumen el criterio de Fierro (2016), al concretar el plan de sistematización en tres aspectos esenciales que son comunes en la bibliografía consultada sobre la temática. Ellos son: 
- Diseño de la sistematización, como el momento en que se realiza la definición del objetivo, la determinación del eje y el objeto de la sistematización, así como la identificación de actores claves de la experiencia a sistematizar

- La recuperación histórica e interpretación crítica de las experiencias y la elaboración de conclusiones (conocido como las «lecciones aprendidas»)

- Elaboración de los productos de comunicación de las experiencias sistematizadas (en este caso, presentación de exigencias básicas para la formación de habilidades informáticas de la programación)

Siguiendo el plan de sistematización propuesto, se determinó el siguiente objetivo de la sistematización:

Reconstruir las experiencias vividas en torno a la formación y desarrollo de habilidades informáticas de la programación a fin de encontrar determinados requerimientos que faciliten dicho proceso, en la formación del profesor de Informática.

El objeto de la sistematización se concreta, de manera general, en las experiencias vividas en la etapa comprendida entre los cursos 2006-2007 hasta el curso 2014-2015 teniendo en cuenta los cambios de contexto en las diferentes etapas de análisis del proceso de formación investigativa el objeto y el eje de la sistematización que varía en correspondencia con ello. Como actores claves que participan de las experiencias que se sistematizan, se encuentran:

- Los grupos de estudio conformados por los estudiantes de las especialidades de Informática, curso Encuentro y Educación Laboral Informática en el curso diurno, en los que se diagnostican los problemas o potencialidades relacionadas con la formación y desarrollo de habilidades informáticas de la programación en cada etapa de la investigación y sobre los que se influye de manera paulatina para determinar los cambios que operan y que determinan, posteriormente, transformaciones en el proceso. Estos grupos se reestructuran en cada una de las etapas de la sistematización en correspondencia con las condiciones en que se desarrolla el proceso de enseñanza aprendizaje de la Programación.

- El grupo focal está integrado por los profesores que forman parte del colectivo que imparte asignaturas de Programación. Este grupo focal se comporta como consultor y evaluador de las experiencias, aportando criterios significativos para la construcción de los resultados que se obtienen. También fue modificado a partir del contexto correspondiente a cada etapa de la sistematización.

Los principales instrumentos y técnicas que sirvieron para el registro y el análisis de la información fueron: la investigación documental, el diario de 
campo, la observación participante, el análisis de los productos del proceso pedagógico, la entrevista a profundidad y el grupo focal. Para el ordenamiento o reconstrucción cronológica de la recuperación histórica, se delimitaron tres etapas:

Primera etapa: La formación y desarrollo de habilidades informáticas de la programación en condiciones de universalización de la Educación Superior (2006-2009)

Segunda etapa: La formación y desarrollo de habilidades informáticas de la programación en el profesional de la especialidad Informática con la centralización de la especialidad (2009-2011).

Tercera etapa: La formación y desarrollo de habilidades informáticas de la programación en el profesional de las especialidades que forman profesores de Informática con transformaciones en los planes de estudio (2011-2015).

El criterio de establecimiento de las etapas atiende a los cambios curriculares producidos y a los provocados en los modelos de formación de los estudiantes de la especialidad Informática.

\section{Resultados y anÁlisis}

\subsection{Primera etapa: La formación y desarrollo de habilidades informáticas de la programación en condiciones de universalización de la Educación Superior (2006-2009)}

Se orienta hacia el diagnóstico del estado de la formación y desarrollo de habilidades en los estudiantes de la carrera de Licenciatura en Educación en la especialidad de Informática. El eje de la sistematización de esta etapa se dirige a la valoración de las necesidades, potencialidades y carencias que inciden en la formación y desarrollo de habilidades informáticas de la programación. Se tomó a las experiencias vividas en esta etapa como objeto de la sistematización.

Durante el periodo 2006-2009, se tomó como grupo de estudio a los 100 estudiantes matriculados entre el 2do y 4 to año en condiciones de universalización de la Educación Superior, por haber recibido al menos una asignatura relacionada con la programación. La interacción con este grupo se realiza con el objetivo de determinar el nivel de desarrollo de las habilidades informáticas de la programación que poseen. Intervienen, además, como grupo focal, los 10 profesores que imparten asignaturas de Programación en las diferentes sedes de la provincia Villa Clara, que actuaron como consultares al caracterizar el proceso de formación y desarrollo de habilidades. Emergen, en este período, las siguientes categorías y subcategorías: 
Categoría: Diagnóstico de los actores claves del proceso

Subcategorías:

Insuficiencias, necesidades y potencialidades en la formación y desarrollo de habilidades informáticas de la programación en los estudiantes de la carrera

Insuficiencias, necesidades y potencialidades de los profesores para dirigir el proceso de formación y desarrollo de habilidades informáticas de la programación

Como miembros del colectivo de profesores que imparte asignaturas de Programación, las autoras comienzan a realizar observaciones participantes a reuniones metodológicas con jefes de sedes, jefes de carrera y profesores a tiempo parcial de la disciplina. Se analizan, además, los informes de promoción de las diferentes sedes y se observan clases en cada una de ellas. Con la aplicación de estos métodos, el empleo del diario de campo y la triangulación metodológica, se revelaron insuficiencias en el proceso de formación y desarrollo de las habilidades informáticas de la programación.

Entre ellas, se destacaron las siguientes:

- Los estudiantes no sabían resolver problemas con el empleo de un lenguaje de programación porque no sabían realizar las acciones que incluye esta habilidad. Estas acciones no son más que habilidades específicas de la programación. Es decir, los estudiantes no sabían resolver problemas porque no sabían elaborar algoritmos, codificar algoritmos o poner a puntos programas.

- Las tareas docentes que se proponían para el desarrollo de las habilidades específicas de la programación no se planificaban de forma gradual y sistemática. Esta situación no propiciaba que los estudiantes tomaran conciencia de los logros que iban alcanzando con su realización.

- Los profesores no tenían un conocimiento acabado acerca de la estructura interna que conforman las habilidades específicas de la programación.

- Los profesores no incorporaban, al proceso de enseńanza aprendizaje, métodos que permitieran al estudiante conocer las vías que puede emplear para dirigir el proceso de formación y desarrollo de las habilidades específicas de la programación en su accionar en la escuela.

Tomando en consideración estas regularidades, se precisó indagar sobre las posibles causas que provocaron las insuficiencias en el proceso de formación y desarrollo de las habilidades informáticas de la programación. Para ello, se entrevistó a profesores que impartían clases en esta disciplina que conforman el grupo focal y a estudiantes que habían recibido, al menos, una de las asignaturas de Programación. Se analizaron, también, cuestionarios de 
evaluaciones y planes de clases. A partir de los resultados de estas entrevistas y de las reflexiones realizadas con el grupo focal, se determina que las principales insuficiencias se centran en la formación de la habilidad, pues no existía un consenso entre los miembros del grupo focal sobre cómo proceder para poder formar una habilidad informática de la programación en un estudiante que no solo debe saber operar con ella, sino que también debe posteriormente poder dirigir este proceso en la escuela.

La habilidad rastrear se determinó como una de las más afectadas. Por la importancia que reviste esta habilidad dentro del sistema, se determinó comenzar a realizar varias acciones que propiciaran la disminución de las insuficiencias que de ella poseían los estudiantes. Con tal motivo, durante el último ańo de esta etapa, se realizó la propuesta de un sistema de acciones para esta habilidad, una tipología de ejercicios para su desarrollo y un software educativo que puede ser empleado como medio de enseńanza en su proceso de desarrollo.

$\mathrm{Al}$ concluir esta primera etapa se revelaron elementos que potenciaban y debilitaban las experiencias vividas. Entre estos se encuentran:

- Se realizó un diagnóstico real y objetivo del proceso de formación y desarrollo de las habilidades informáticas de la programación, determinando varias insuficiencias.

- Se logró sensibilizar a los actores del proceso vivido (grupos de estudio y grupo focal) en cuanto a la necesidad de favorecer el proceso de formación y desarrollo de las habilidades informáticas de la programación.

- Se conformó un sistema de acciones para una de las habilidades informáticas de la programación (rastrear).

- Se comprobó la tendencia al empleo de métodos problémicos que favorecen la formación y desarrollo de la habilidad resolver problemas con el empleo de un lenguaje de programación en el proceso de enseńanza aprendizaje de la disciplina.

- Persistían las insuficiencias en el desarrollo de la habilidad rastrear y el resto de las habilidades informáticas de la programación.

- Se mantuvo la falta de motivación de los estudiantes por las asignaturas de la disciplina.

- Se evidenció la falta de preparación de los profesores para dirigir el proceso de formación y desarrollo de las habilidades informáticas de la programación.

Como lecciones aprendidas de esta etapa, se revela la necesidad de que el análisis del proceso de formación de habilidades informáticas de la programación, más que en los sujetos, se centre en el proceso en sí, lo que 
constituye un hallazgo dentro de la investigación. A partir de ello, se revelan las siguientes necesidades:

- Emplear de métodos problémicos en el proceso, que demuestren la necesidad de adquirir una habilidad informática de la programación y destaque la significación que pueda tener para la vida del estudiante durante su carrera y como futuro profesional

- Contar con un sistema de acciones y operaciones que conforme la estructura interna de la habilidad que se desea formar

- Contar con procedimientos que permitan formar las habilidades informáticas de la programación, de manera tal que el estudiante adquiera no solo las acciones que las conforman, sino también las vías metodológicas para, posteriormente, poder dirigir este proceso

\subsection{Segunda etapa: La formación y desarrollo de habilidades informáticas de la programación en el profesional de la especialidad Informática con la centralización de la especialidad (2009-2011)}

Esta etapa se distingue por la necesidad de aproximación a formas de proceder para facilitar la actuación de los actores del proceso a partir del estado actual determinado en el diagnóstico general. Por tanto, el eje de sistematización está dirigido a la determinación de procederes metodológicos que favorecen el proceso de formación de las habilidades informáticas de la programación.

A partir del curso 2009-2010, se comienza un proceso de actualización de la carrera Informática. Se agrupan, paulatinamente, las diferentes sedes municipales en una sede central. Se mantiene como grupo de estudio a los estudiantes matriculados entre el 2do y 4to año de la carrera Licenciatura en Educación en la especialidad de Informática, aunque disminuye su cifra en consonancia con la reducción de la matrícula. Por esta razón, el grupo de estudio solo es conformado por 70 estudiantes, atendiendo al mismo criterio que en la etapa anterior. El claustro a tiempo parcial también disminuye por las razones antes expuestas. Dos de las autoras comienzan a impartir clases en grupos de tercer año de la especialidad y la otra lo hace en un grupo de segundo año.

En este período, el grupo focal se conforma por los cuatro profesores que imparten asignaturas de Programación que laboran en el departamento de Informática a tiempo completo y dos profesores a tiempo parcial que complementan el claustro de la sede central. Este grupo aportó ideas relevantes en el proceso investigativo seguido. Durante el propio curso 2009-2010, la autora principal asume la dirección de la disciplina de Programación, lo que facilitó el trabajo metodológico en este nivel estructural. 
En esta etapa, se mantiene la categoría: diagnóstico de los actores claves del proceso, pero se transforma obteniéndose como subcategoría los logros, insuficiencias y tendencias en los actores claves del proceso a partir de los procederes que se van introduciendo en el proceso de formación y desarrollo de las habilidades informáticas de la programación. En este sentido, se utiliza el grupo de estudio para caracterizar dicho proceso, a partir de la incorporación de procederes metodológicos dirigidos a la formación y desarrollo de estas habilidades. El grupo focal se emplea, en esta etapa, en sus dos variantes como evaluadores de los procederes que se elaboran y como consultores para evaluar los logros y dificultades que se van obteniendo en la práctica escolar.

Emerge, además, en este período, la categoría: proyecciones en el orden teórico sobre el proceso de formación y desarrollo de habilidades informáticas de la programación. Como subcategoría, se determina la aproximación a la determinación de exigencias básicas a emplear en la dirección del proceso de formación y desarrollo de habilidades informáticas de la programación.

Las autoras comenzaron a poner en práctica los resultados obtenidos en investigaciones precedentes sobre un sistema de acciones y operaciones que conforman las habilidades informáticas de la programación que se trabajan en la disciplina. En tal sentido, los criterios del grupo focal fueron determinantes, incluso para la obtención final del sistema de acciones y operaciones con que se operó en cada asignatura.

Como parte del trabajo metodológico de la disciplina, se desarrollaron, además, una serie de talleres en los que se debatieron los requerimientos a tener en cuenta en el proceso de formación de habilidades informáticas de la programación. Para evaluar el impacto del sistema de talleres, se emplearon, como métodos, la observación participante, la entrevista a los estudiantes de la sede central como grupo de estudio y el grupo focal que aplicaba, en sus clases, los elementos debatidos en los talleres. Se analizaron, también, los resultados obtenidos en los exámenes efectuados al grupo de estudio donde se medía el desarrollo de habilidades informáticas de la programación.

A partir de la triangulación metodológica, se concluye que los profesores poseen un mayor conocimiento del sistema de acciones y operaciones que conforman las habilidades informáticas de la programación. También, se constató que aplican, en sus clases, métodos problémicos que permiten demostrar la necesidad de adquirir la nueva habilidad que se desea formar. De esta manera, se destaca la significación que puede tener para los estudiantes en su vida y se logra una mayor motivación de estos por las asignaturas de la disciplina.

Resultó un elemento importante que emergió de este proceso: la necesidad de que, al presentar la habilidad que se desea formar, se presente su sistema de acciones 
haciendo énfasis en las habilidades lógicas e informáticas que la conforman. De igual manera, se comprobó la necesidad de que la ejercitación que se planifique para desarrollar las habilidades informáticas de la programación se caracterice por su variedad y graduación, de manera que le permita al estudiante realizar un mismo tipo de acción, pero que no cree estereotipos en él. Con el objetivo de favorecer esta ejercitación, las autoras, como parte del grupo focal, elaboraron tipologías de ejercicios tomando en cuenta el sistema de acciones de las habilidades informáticas de la programación asumido para la investigación. Durante este período, se constataron, además, resultados favorables en el desarrollo de las habilidades informáticas de la programación que repercuten en un mayor desarrollo de la habilidad resolver problemas con un lenguaje de programación.

La socialización de las experiencias obtenidas en esta etapa, debatidas con el grupo focal, sientan pautas para la elaboración de aproximaciones teóricas que conllevan a la necesidad de establecer relaciones entre las habilidades informáticas de la programación y el resto de las habilidades que debe formar el estudiante (lógicas, informáticas e informáticas de la programación). Por otra parte, se destaca que aún no se han revelado formas de actuación de los estudiantes en su accionar en la escuela, que se correspondan con la dirección de este proceso.

Entre las lecciones aprendidas al final de esta etapa, se precisan las siguientes:

- Se hace necesario buscar métodos que permitan establecer relaciones entre las habilidades informáticas de la programación y el resto de las habilidades que debe formar.

- Se precisa proponer a los estudiantes las vías que deben seguir para formar y desarrollar esta habilidad en la escuela.

- Resulta importante el empleo de tipologías de ejercicios que favorezcan el desarrollo del sistema de acciones de la habilidad recién formada.

- Como una exigencia para la formación de habilidades informáticas de la programación, debe existir una unidad entre las habilidades lógicas, informáticas, informáticas de la programación y los fundamentos de la enseñanza problémica.

\subsection{Tercera etapa: La formación y desarrollo de habilidades informáticas de la programación en el profesional de las especialidades que forman profesores de Informática con transformaciones en los planes de estudio (2011-2015)}

El elemento distintivo de esta etapa lo constituye la reflexión teórica sobre las vías metodológicas a emplear en el proceso de formación de habilidades informáticas de la programación en la formación del profesor de Informática. 
De ahí que, teniendo como objeto las experiencias vividas entre los años 2011 y 2015, se determina como eje de sistematización la búsqueda de exigencias básicas para favorecer el proceso de formación y desarrollo de habilidades informáticas de la programación en la formación del profesor de Informática.

Durante esta etapa se reducen a 30 los estudiantes que reciben las asignaturas de Programación, bajo un plan de estudio C modificado. No existen transformaciones significativas en el comportamiento de los actores claves del proceso a partir de los procederes que se van introduciendo, por lo que no es necesario continuar profundizando en su diagnóstico. El objetivo de la interacción con el grupo de estudio, en esta etapa, se centra en la obtención de nuevas exigencias para desarrollar el proceso de formación y desarrollo de habilidades informáticas de la programación.

Como resultado de la etapa anterior, emergen nuevas categorías, cada una de ellas con sus respectivas subcategorías:

Categoría: Fundamentos teóricos para la dirección del proceso de formación y desarrollo de habilidades informáticas de la programación en la formación del profesor de Informática. Subcategoría: Determinación de exigencias que constituyen sustento teórico para el empleo de los procederes determinados.

Categoría: Resultados obtenidos. Subcategorías: Valoración de las tendencias durante el proceso de formación y desarrollo de habilidades informáticas de la programación en el estudiante, como resultado del proceder elaborado.

A pesar de implementarse, en esta etapa, el plan $\mathrm{C}$ modificado, la disciplina de Programación no sufre cambios significativos. Solo se incrementan las horas clases en dos de las asignaturas, por lo que resulta válida la sistematización de los resultados obtenidos con relación a la formación de habilidades informáticas de la programación.

En esta etapa, la dirección del trabajo metodológico de la disciplina continúa a cargo de las autoras, mientras que el grupo focal se reduce solo a los cuatro profesores a tiempo completo de la disciplina del departamento, pues dejan de impartir docencia los profesores a tiempo parcial.

Durante este período, se desarrollaron talleres, con el grupo focal, que precisaban cómo presentar la habilidad informática de la programación que se desea formar, por lo que se desempeñan fundamentalmente como consultores. Del análisis con el grupo focal, se deriva la necesidad de determinar la relación existente entre la habilidad a formar y el sistema de conocimientos. Para ello se debe tener presente:

- La estructuración del sistema de conocimientos en núcleos temáticos conceptuales 
- Analizar el sistema de acciones y operaciones que conforman la habilidad: El análisis debe permitir a través del ejemplo y la explicación, que el estudiante conozca qué hacer para realizar cada una de las acciones de la habilidad, indicando cuáles son las operaciones necesarias y suficientes para ello y cómo poder controlarlas, así como la relación que posee con el sistema de conocimientos de la asignatura.

- Determinar la relación existente entre la habilidad y el resto de las habilidades del sistema: Este análisis debe hacerse de manera bidireccional, destacando el lugar que ocupa la habilidad a formar dentro del sistema, particularmente con el sistema de habilidades informáticas de la programación, a partir del sistema de acciones que la conforman; así como dela importancia que reviste la nueva habilidad para cumplir con los objetivos propuestos.

- Evaluar el proceso seguido en la formación y desarrollo de la habilidad, resultando importante no solo el conocimiento que el profesor logre acerca de cómo se ha formado la habilidad en cada estudiante, sino también de la propia autoevaluación que realice el estudiante sobre el proceso

Emergen, de este momento de la sistematización, dos exigencias básicas para dirigir el proceso de formación y desarrollo de habilidades informáticas de la programación:

- Unidad entre las habilidades lógicas, informáticas, informáticas de la programación y los fundamentos de la enseñanza problémica

- Formalización del nivel de interrelaciones existentes entre las habilidades informáticas de la programación, teniendo en cuenta los sistemas de acciones que las conforman y las vías metodológicas empleadas para su formación

Durante todo el período, se realizan observaciones participantes a las clases de la disciplina. Se realizan, también, entrevistas al grupo focal y al grupo de estudio que permitieron determinar, al final de la investigación, los siguientes resultados:

- Los profesores de la disciplina se apropiaron de métodos particulares para la formación y desarrollo de las habilidades informáticas de la programación, a partir de las exigencias propuestas.

- Los estudiantes muestran su motivación por las asignaturas de la disciplina, basado en la significación que tienen para ellos los contenidos tratados.

- Existe un mayor conocimiento, tanto por parte de los profesores como de los estudiantes, del sistema de acciones que conforman las habilidades informáticas de la programación, así como de las relaciones que ellas poseen entre sí y las existentes con el resto de los sistemas de habilidades. 
- Durante el proceso de formación de las habilidades informáticas de la programación, el estudiante no solo forma la habilidad, sino que adquiere vías para poder dirigir posteriormente este proceso en la escuela.

- Existe un mayor desarrollo de las habilidades informáticas de la programación, que repercute favorablemente en el desarrollo de la habilidad resolver problemas con el uso de un lenguaje de programación.

Al concluir el período tomado como referente para la realización de la sistematización de experiencias vividas, se efectuó una interpretación crítica de los resultados obtenidos. El análisis de los hallazgos encontrados y de las lecciones aprendidas en cada etapa permite afirmar que el tránsito por cada una de ellas posibilitó determinar las necesidades, potencialidades y carencias que inciden en la formación y desarrollo de habilidades informáticas de la programación, así como la determinación de procederes y exigencias metodológicas que favorecen este proceso. Entre los principales hallazgos, se destaca la necesidad de considerar la unidad existente entre las habilidades lógicas, informáticas, informáticas de la programación y los fundamentos de la enseñanza problémica. Resulta importante, además, formalizar el nivel de interrelaciones existentes entre las habilidades informáticas de la programación, a partir de los sistemas de acciones que las conforman, y concebir la ejercitación con el uso armónico de tipologías de ejercicios que faciliten la sistematización de dichas acciones. No se debe perder de vista el planteamiento de problemas que le permitan al estudiante adquirir, de manera consciente, no solo el sistema de acciones de las habilidades informáticas de la programación, sino también los procederes metodológicos que puede emplear para dirigir su proceso de formación y desarrollo.

\section{CONClusiones}

La sistematización de experiencias vividas en relación con la formación inicial del profesor de Informática, apartir del curso 2006-2007 hasta el 2014-2015, permitió, con la ayuda de los grupos focales y de estudio, descubrir elementos teóricos y prácticos que conllevaron a la elaboración de procederes y exigencias que pueden favorecer dicho proceso.

Como resultado de la sistematización de experiencias, se logró que los profesores de la disciplina se apropiaran de un sistema de acciones para cada una de las habilidades informáticas de la programación que deben formar y desarrollar, así como de los métodos necesarios para ello. Además, los estudiantes alcanzaron avances en el desarrollo de dichas habilidades, así como en la obtención consciente de las vías metodológicas para dirigir, posteriormente, dicho proceso en la escuela. 
Las experiencias vividas, así como los procederes y exigencias básicas para la formación y desarrollo de habilidades informáticas de la programación en la formación de profesores de Informática obtenidas a partir de la sistematización descrita anteriormente, se han incorporado como aportes didácticos distintivos que caracterizan este proceso. Por tal razón, se considera que han constituido punto de partida y basamento teórico para el desarrollo del proceso de enseñanza aprendizaje de las asignaturas de Programación en los nuevos planes de estudio de esta especialidad. Además, se puede afirmar que la sistematización proporcionó sugerencias, intuiciones, pistas, provocaciones, que incentivaron otras experiencias e investigaciones relacionadas con la resolución de problemas en Programación, sobre la base del intercambio de aprendizajes ya interpretados.

\section{REFERENCIAS BIBLIOGRÁFICAS}

Aguilasocho, D. (2004). Propuesta metodológica para la enseñanza de la programación en el bachillerato mexicano. (Tesis doctoral). Instituto Superior Pedagógico «Félix Varela Morales», Santa Clara, Cuba.

Alea, M., Miqueo, J. y Aguiar, A. (2005). Consideraciones sobre las habilidades fundamentales en la enseñanza de la informática. Universidad del Deporte Nancy Uraña Romagosa. V Conferencia Internacional Científico Pedagógica de Educación Física y Deportes.

Álvarez, C. (1992). Didáctica: la escuela en la vida. La Habana: Pueblo y Educación.

Báez, J. y Onrubia, J. (2016). Una revisión de tres modelos para enseñar las habilidades de pensamiento en el marco escolar. Perspectiva Educacional. Formación de Profesores, 55(1), 94-113. Recuperado de http://www.perspectivaeducacional.cl

Coloma, C. R. y Tafur, R. M. (2000). Sobre los estilos de enseńanza y de aprendizaje. Educación, $I X(17)$, 51-79. Recuperado de http://revistas.pucp.edu. pe/index.php/educacion/article/view/5255/5249

Díaz, K. I. (2013). Las habilidades informáticas de la programación en la formación inicial del profesor de la especialidad Informática. (Tesis doctoral). Universidad de Ciencias Pedagógicas «Félix Varela», Santa Clara, Cuba.

Díaz, K. I. y Crespo, T. (2010). La conceptualización de las habilidades informáticas. Revista electrónica IPLAC, No.5. Recuperado de http://www.revista. iplac.rimed.cu

Expósito, C., Cruañas, J., Gener, E.J., de la Noval, N., Rivero, A. y Peñalver, L. (2001). Algunos elementos de metodología de la enseñanza de la informática. Ciudad de La Habana: Pueblo y Educación. 
Ferreira, A. y Rojo, G. (2006). Enseñanza de la programación. Revista Iberoamericana de Tecnología en Educación y Educación en Tecnología., I(1), 1-8. Recuperado de http://www. teyet-revista.info.unlp.edu.ar

Fierro, E. R. (2016). Utilización de la analogía en la resolución de problemas en el proceso de enseñanza-aprendizaje de la Programación. (Tesis doctoral). Universidad Central Marta Abreu de Las Villas, Santa Clara, Cuba.

Fischer, P. (2011). Theaching programming to beginners. Recuperado de http:// www.2.imm.dtu.dk

Jackson, D. y Miller, R. (2009). A New Approach to Teaching Programming. Recuperado de http://people.csail.mit.edu

López, M. (1990). ¿Sabes enseñar a describir, definir, argumentar? La Habana: Pueblo y Educación.

Matthiasdóttir, Á. (2011). How to teach programming languages to novice students? Recuperado de http://ecet.ecs.ru.acad.bg

Murillo, M. (2006). Explorando el proceso de enseñanza y de aprendizaje en el área de la programación de computadoras. Revista electrónica Actualidades Investigativas en Educación, 6(1), 1-28.

Navarro, A. y Roche, A. M. (2013). Investigación en educación: los procesos de sistematización de experiencias educativas. Oficios Terrestres, 1(29). Recuperado de http://perio.unlp.edu.ar

Sánchez, J. (2007). Apuntes de fundamentos de programación. Recuperado de http://lawebdelprogramador.com

Suárez, F. (2008). Modelo de enseñanza de la programación desde su dimensión didáctica basado en CUPI2. Recuperado de http://fjsuarezv.blogspot.com

Van de Velde, H. (2008). La sistematización de experiencias educativas: un espacio para la reflexión crítica y la transformación de la práctica. Managua, Nicaragua: IDE-UCA.

Vargas, C. M. (2014). Estrategias didácticas para el desarrollo de la identidad cultural en educación primaria. Educación, XXIII(45), 25-50. Recuperado de http://revistas.pucp.edu.pe

Vasconcelos, J. (2007). Basic strategy for algorithmic problem solving. Recuperado de http://www.cs.jhu.edu 